\title{
Dupuytren's Contracture with Secondary Ulnar Neuropathy
}

\author{
Chen Fei $\mathrm{Ng}^{1}$ \\ ${ }^{1}$ Neurology Unit, Department of Medicine, Universiti Kebangsaan \\ Malaysia Medical Centre, Cheras, Kuala Lumpur, Malaysia
}

\begin{abstract}
Address for correspondence Chen Fei Ng, MD, MRCP, Neurology Unit, Department of Medicine, Universiti Kebangsaan Malaysia Medical Centre, Jalan Yaacob Latif, Bandar Tun Razak, 56000 Cheras, Kuala Lumpur, Malaysia (e-mail: n.chenfei@gmail.com).
\end{abstract}

\author{
Abstract \\ Keywords \\ - Dupuytren's disease \\ - Dupuytren's \\ contracture \\ - ulnar neuropathy \\ - neurophysiology
}

\begin{abstract}
Dupuytren's disease is a progressive fibrotic condition of the hand. The underlying pathomechanism is not fully known. Dupuytren's contracture can be seen in patients with diabetes mellitus, chronic alcoholism, smoking, or hand trauma. It is uncommon to affect the neurovascular supply causing clinical symptoms. We describe a patient with idiopathic Dupuytren's disease complicated with bilateral ulnar neuropathies and highlight the importance of such rare treatable complication.
\end{abstract}

\section{Introduction}

Dupuytren's disease is a common fibroproliferative condition affecting the palmar and digital fascia. The prevalence of Dupuytren's disease is higher in chronic alcoholic drinker. However, there is no clear causal relationship and there are many other factors that may predispose to the condition. The focal pathological process in the hands may potentially cause structural compression, in severe cases, leading to symptomatic neuropathy if surrounding neurovascular bundle is compromised.

\section{Case Description}

A 63-year-old man presented to neurology clinic with bilateral hand numbness and weakness for 6 months. The symptoms were preceded by a 5-year history of slowly progressive contracture of his fingers bilaterally. He had long-standing type 2 diabetes mellitus and hypertension for more than 10 years. There was no significant family history of note and he did not smoke or drink alcohol. On examination, there were mild wasting of the hypothenar eminences and interossei guttering. In addition, there were striking flexion deformity and clawing involving his fingers on the right hand, less so on the left hand ( $\boldsymbol{- F i g .} \mathbf{1})$. The muscle power of the abductor digiti minimi (ADM) was Medical Research Council grade $2 / 5$ on the right and $3 / 5$ on the left, and the first dorsal interosseous (FDIO) was grade $3 / 5$ bilaterally. The muscle power of abductor pollicis brevis, flexor digitorum superficialis, and profundus were normal. The biceps, triceps, and supinator jerks were present bilaterally. On sensory examination, pinprick sensation was reduced on the palmar aspect of the fourth and fifth fingers but normal on the dorsal surface bilaterally. The median and radial dermatomal distributions were spared. He had otherwise no stigmata of chronic liver disease. Routine blood tests for complete blood count, liver function test, and erythrocyte sedimentation rate were normal. Nerve conduction study and needle electromyography revealed active and chronic neurogenic changes in the ulnar-innervated muscles distal to the wrist with demyelinating and axonal features, much worse on the right side (-Tables 1 and 2). He was diagnosed with bilateral distal ulnar neuropathies secondary to Dupuytren's contracture and surgical release was subsequently performed.

\section{Discussion}

The presenting complaints of sensory and motor symptoms with claw hands led to the diagnosis of bilateral ulnar neuropathies evident on neurophysiology. The bilateral ulnar sensory studies showed prolonged latency and conduction slowing, with dispersed compound muscle action potential 


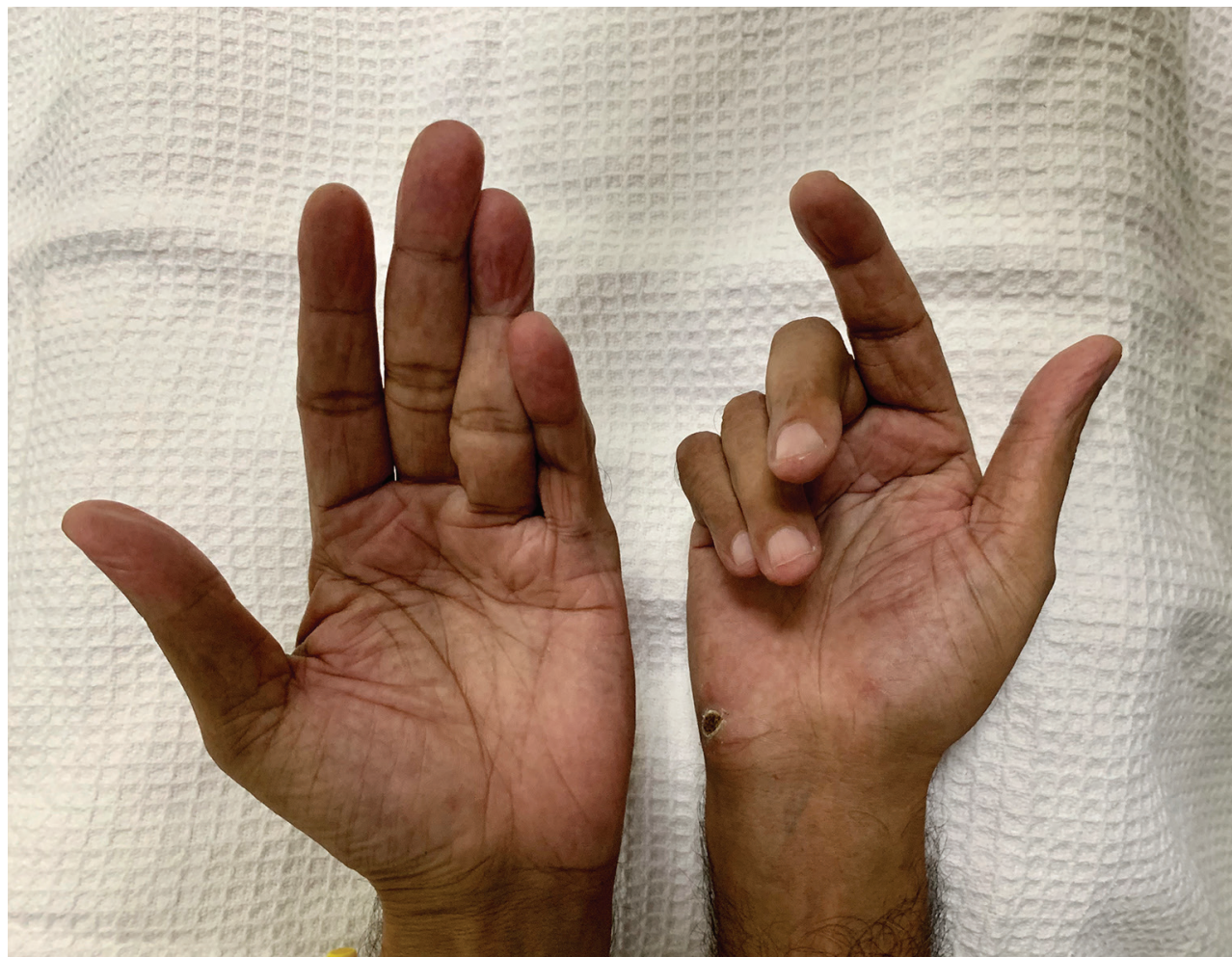

Fig. 1 There was flexion deformity of the middle, ring, and little fingers with prominent wasting of the abductor digiti minimi muscle on the right side. On the left hand, palmar thickening and fibrotic cord were seen over the fourth metacarpophalangeal joint.

Table 1 Nerve conduction study of the upper limbs

\begin{tabular}{|c|c|c|c|c|}
\hline Sensory nerve study & Recording site & Latency, ms & Amplitude, $\mu \mathrm{V}$ & Velocity, $\mathrm{m} / \mathrm{s}$ \\
\hline L median & Digit II & 3.20 & 28.7 & 49 \\
\hline R median & Digit II & 2.94 & 25.2 & 53 \\
\hline L ulnar & Digit V & 4.58 & 11.2 & 32 \\
\hline Rulnar & Digit V & 4.38 & 6.2 & 29 \\
\hline L dorsal ulnar cutaneous & Dorsal hand & 1.89 & 12.4 & 52 \\
\hline L dorsal ulnar cutaneous & Dorsal hand & 2.01 & 14.3 & 48 \\
\hline Motor nerve study & Recording site & Latency, ms & Amplitude, mV & Velocity, $\mathrm{m} / \mathrm{s}$ \\
\hline R median-wrist & APB & 3.8 & 11.4 & \\
\hline R median-elbow & APB & 8.2 & 10.4 & 52 \\
\hline L ulnar-wrist & ADM & 3.44 & 11.3 (dispersed) & \\
\hline L ulnar-below elbow & ADM & 9.01 & 9.5 (dispersed) & 54 \\
\hline R ulnar-wrist & ADM & 3.65 & 9.9 (dispersed) & \\
\hline R ulnar-below elbow & ADM & 9.27 & 9.0 (dispersed) & 53 \\
\hline F wave (min) & & Latency, ms & & \\
\hline L ulnar & & 34.74 & & \\
\hline Rulnar & & 36.51 & & \\
\hline
\end{tabular}

Abbreviations: ADM, abductor digiti minimi; APB, abductor pollicis brevis; L, left; $R$, right.

response on ulnar motor studies. These findings were highly suggestive of focal demyelination in keeping with structural compression. In addition, needle electromyography revealed active denervation changes in the right ADM and FDIO and chronic reinnervation in the bilateral ADM and FDIO indicating axonal loss. The normal studies for dorsal ulnar cutaneous nerve and flexor carpi ulnaris further localized the lesions distal to the wrists.

The presence of fibrotic cords and contracture clinched to the underlying etiology of Dupuytren's disease. Dupuytren's disease is a fibroproliferative condition of the hands. Although the pathogenesis is not fully understood, it was 
Table 2 Needle electromyography of the upper limbs

\begin{tabular}{|l|l|l|l|}
\hline Muscle & Spontaneous activity & MUAP & Recruitment pattern \\
\hline L first dorsal interosseous & None & Mildly prolonged and polyphasic & Mildly reduced \\
\hline L abductor digiti minimi & None & Mildy polyphasic & Mildly reduced \\
\hline L abductor pollicis brevis & None & Normal & Normal \\
\hline R first dorsal interosseous & Positive sharp waves & Prolonged and polyphasic & Reduced \\
\hline R abductor digiti minimi & Positive sharp waves & Prolonged and polyphasic & Reduced \\
\hline R abductor pollicis brevis & None & Normal & Normal \\
\hline R flexor carpi ulnaris & None & Normal & Normal \\
\hline R extensor indicis proprius & None & Normal & Normal \\
\hline
\end{tabular}

Abbreviations: L, left; MUAP, motor unit action potential; R, right.

suggested to be due to local hypoxia and chronic ischemia. Factors such as alcohol, smoking, diabetes mellitus, and certain anticonvulsants are commonly associated with Dupuytren's disease. ${ }^{1}$ Interestingly, ulnar neuropathy is not a recognized association. In Dupuytren's disease, the normal fascial bands in the hand become fibrotic cord which may involve palmar, palmodigital, or digital regions. Pathological examination showed that digital cord arising from the ADM muscle may lead to entrapment and displacement of the underlying neurovascular bundle. ${ }^{2}$ The local compression, in addition to the predisposition of underlying microvascular disease due to diabetes has most likely resulted in secondary ulnar neuropathy. This association is rarely seen but important to recognize as prompt surgical release may prevent severe neuropathy and functional loss.

\section{Funding}

None.

\section{Conflict of Interest}

None declared.

\section{References}

1 Townley WA, Baker R, Sheppard N, Grobbelaar AO. Dupuytren's contracture unfolded. BMJ 2006;332(7538):397-400

2 Rayan GM. Dupuytren disease: anatomy, pathology, presentation, and treatment. J Bone Joint Surg Am 2007;89(1):189-198 